\title{
Enhanced Data Services in GPRS Networks via Auction-Based Prices for Admission
}

\author{
Saravut Yaipairoj and Fotios C. Harmantzis \\ Telecommunications Management, Stevens Institute of Technology, \\ Castle Point on the Hudson Hoboken, NJ 07030, USA \\ \{syaipair, fharmant\}@stevens.edu
}

\begin{abstract}
We propose an auction-based pricing scheme for admission in a GPRS network, that could enhance the GPRS data service. Important features of our approach are the partitioning of dedicated channels for enhanced data service and the employment of an auction mechanism based on revenue-maximizing auctions (optimal auctions).
\end{abstract}

\section{Introduction}

GPRS data service fails to support most real-time applications, because of its low data throughput. The main cause is due to the resource-sharing between voice and data traffic 3 . Since voice has priority over data, there are only few traffic channels available for data traffic. To be specific, GPRS traffic can capture approximately one or two traffic channels out of eight channels during data transmission. Performance could be even worse during the congestion time. Therefore, it is quite difficult for GPRS to support real-time data services, as long as the problem of resource sharing between voice and data remains. In [1, Odlyzko has proposed a simple but effective scheme that allows Internet users to experience better quality of service, namely, Paris Metro Pricing (PMP). He proposed partitioning of networks and pricing them differently. His technique allows users to experience a better quality of service in the higher-priced network. Even though PMP has not been implemented yet in real networks, since Internet bandwidth has become less expensive, we argue that it could be used in bandwidth-limited IP based network, such as GPRS. To enhance the GSM/GPRS service, we could partition the traffic channels and apply different prices to each group of channels. Since our focus is enhancing the data service, we argue that higher price channels should be dedicated for data service. By doing this, we could lose the efficiency of spectrum usage and degrade both voice and GPRS service in the lower price channel group. To compensate for the potential lost of revenue from channel partitioning, the dedicated channels for "enhanced" GPRS service must be limited and only allocated to users who value them most. Therefore, instead of adopting simple pricing policies for channels, we argue that auction-based prices would be more suitable [2]. In this paper, we propose auction-based pricing for admission to enhance GPRS services. The merits of our proposed model are: a) channel partitioning scheme for GSM/GPRS traffic channels; b) introduction of 
an auction mechanism for enhancing GPRS services; and c) the proposed model takes into account the value distribution of bidders for revenue maximization.

\section{Auction-Based Pricing Model for Admission}

In our model, we assume that GPRS users are offered two types of GPRS services: traditional and enhanced services. In traditional GPRS service, GPRS traffic uses the residual channels left from voice traffic while the enhanced GPRS service uses their own dedicated channels. The user who chooses the enhanced service is called a "heavy" user, which refers to his potentially high volume of their traffic in his call requests. To assure that the dedicated channels are allocated to the users who value them most, we employ auction-based prices to admit those users to the dedicated channel (in contrast to PMP, which employs higher prices). Auction rounds are established periodically. Mobile terminals monitor auction information, such as the beginning and the end of an auction, from a base station through the GPRS attached procedure. Users, who are willing to participate in an auction, submit their bids with their call requests through a Packet Data Protocol (PDP) Context Activation. They are required to bid greater or equal to a reservation price, which is the minimum price assigned by the network [4. The winners will be granted transmission by receiving IP address and their data traffic is allowed to use dedicated channels. Since the auction technique is simply used for admitting users to the dedicated channels, the data services are still based on best-effort. However, users can expect better services because of the fact that the data calls would be able to capture relatively more timeslots in dedicated channels than they do in the existing GPRS networks, where resource sharing of voice and data exists. Note that we can reduce user intervention in the auction process by employing agent programs to monitor auctions and automatically submit bids according to user bidding profile, which basically the pattern of bids of users in the current and the next auction rounds (in case it is a losing bid in the first round).

\subsection{Assigning Dedicated GPRS Channels}

Due to prioritization of voice calls over data calls, the voice blocking probability in GSM/GPRS networks could be approximately determined by the erlang-B formula. However, the number of traffic channels available for voice traffic is now subtracted by the number of dedicated channels. The smaller number of channels for voice traffic would increase the voice blocking probability. Therefore, we need to justify the number of dedicated data channels that should be taken from total traffic channels in order to minimize excessive voice blocking probability. Apparently, the suitable number of dedicated channels depends heavily on the volume of voice calls in the network; we suggest this number should change based on the voice traffic load during the day. 


\subsection{Maintaining High Throughput}

In this subsection, we focus on the performance of the dedicated traffic channel for enhanced data service. It is obvious that each new heavy users in the dedicated channels would impose traffic load that affect the transmission rate of ongoing heavy users. The number of heavy users admitted to the system needs to be limited in order to ensure the welfare of ongoing data transmission in the dedicated channels. We first measure the performance degradation that indicates the excessive number of users. The performance degradation can be measured in terms of packet delay. We need to determine a mechanism that allows us to discourage heavy users from entering the system when the average delay is excessive. In auction theory, the parameter used to constrain the amount of bidders participating in an auction is the auction reservation price [2]. We employ the reservation price to discourage users from participating in the auction when there are too many users already in the system. The change in reservation price should correspond to capacity of dedicated channels at that particular time, as well as the additional packet delay imposed on ongoing heavy users. The reservation price in an auction starts with a default number, then, price can be increased or decreased in later rounds, based on a target packet delay determined by the operator of the system.

\subsection{Optimal Auctions}

Our auction-based pricing model is implemented at the admission control level for heavy users. The auction is a multi-unit second-price sealed bid auction or Vickery auction [2]. To maximize revenue gained from the auction, we employ the revenue-maximizing auction or so-called optimal auction [4. The optimal auction in this case is basically a Vickery auction with an introduction of a reservation price. Let us assume that the bidders' private value is independently and identically distributed (IID) drawn from the common distribution $F(v)$ : where $F(\underline{v})=0, F(\bar{v})=1$, and $F(v)$ strictly increasing and differentiable over the interval $(\underline{v}, \bar{v})$. We assume further, all bidders are risk neutral. ¿From the revenue equivalent theorem derived by Riley and Samuelson [4, the common equilibrium bidding strategy for Vickery auction yields an expected revenue to the seller as follows:

$$
E=n \int_{v_{*}}^{\bar{v}}\left(v F^{\prime}(v)+F(v)-1\right) F(v)_{n-1} d v
$$

where $v_{*}$ is the reservation price, $n$ is the number of bidders and $\bar{v}$ is the maximum bidders' value. Even though the revenue equivalent theorem is used to analyze the auction of a single item, the theorem can be generalized for our auction. The auction in our case is a multi-unit auction in which each winner would require only one unit, i.e. the right to access dedicated channels. Hence, we can use the generalization of Vickery auctions, i.e. the $k$ highest bidders would pay the value of the highest losing bid. The revenue-equivalence theorem still holds for these auctions [2]. The reservation price needs to correspond to the private 
valuation of the network resource. The valuation consists of a channel cost and a congestion cost. A channel cost refers to the cost of acquiring dedicated channels from existing GPRS channels, i.e., the opportunity cost for partitioning channel. A congestion cost refers to the cost of additional packet delay for admitting heavy users to the dedicated channels. Based on [4], by making the IID assumption and consider risk-neutral bidders, the reservation price, $v_{*}$, that maximizes the expected revenue satisfies

$$
\begin{array}{r}
v_{*}=v_{0}+1-\frac{F\left(v_{*}\right)}{F^{\prime}\left(v_{*}\right)} \\
v_{0}=A+C \cdot T
\end{array}
$$

where $v^{*}$ is the optimal reservation price, $v_{0}$ is the private valuation of the network. $A$ is the channel cost as described earlier; $C$ is a delay-cost factor representing the relationship between the delay in dedicated channels and cost imposed into the system; and $T$ is the average packet delay of the system. The underlined assumption is that additional users would yield additional traffic load in the system, which results in an increase of system delay. These cost need to be added into the private valuation of the network in order to reflect the real cost of the data service. Equations 1 and 2 provide very important parameters for our auction design. Note that the reservation price calculated from equation 2 is independent of the number of bidders [2. Therefore, we can maximize revenue created from the auction model with the minimum number of bidders.

\section{Conclusion}

In this paper, we have proposed an auction mechanism, which can provide superior data services in GPRS networks. The approach to our proposed mechanism consists of channel partitioning technique and auction mechanism as an admission control of our system.

\section{References}

1. Odlyzko, A. M.,: Paris Metro Pricing: The minimalist differentiated services solution. In: Proc. 1999 7th International Workshop on Quality of Service (IWQoS'99) (1999) 159-161

2. Courcoubetis, C., Weber, R.,: Pricing Communication Networks: Economic, Technology and Modeling. Wiley (2003)

3. Araujo, H., Costa, J., Correia, M.L., Analysis of a Traffic Model for GSM/GPRS. IEEE International Symposium on Personal, Indoor and Mobile Radio Communications, vol.1 (2001) C-124-C-128

4. Riley, J., Samuelson, W.,: Optimal auctions. The American Economic Review, vol.71, issue 3, June (1981) 381-392 